\title{
El buen gobierno de los servicios de salud de producción pública: ideas para avanzar
}

\author{
Good governance of publicly-produced health services: \\ ideas for moving forward
}

José-M anuel Freire ${ }^{1}$ Jose Ramon Repullo ${ }^{2}$
${ }^{1}$ Departamento deSalud Internacional, Escuela Nacional deSanidad, Instituto deSalud Carlos III, Ministerio de Ciencia e Innovación. Av. M onfortede Lemos 5 ES-28006 Madrid España. jmfreire@isciii.es ${ }^{2}$ Departamento de Planificación y Economía de la Salud, Escuela N acional deSanidad, Instituto de Salud Carlos III, M inisterio deCienciaelnnovación, España.
Abstract The good performance of publicly-produced health services is of vital importance, well beyond the health sector. Taking into account the great complexity of the health services in the public sector due both to their public and professional nature, we identify seven Gordian Knots as being responsible for the most frequent problems of publicly produced health services in Spain and Latin America. From the concept of good governance we take its character as a normative and ethical benchmark and its potential to renew and invigorate the government of the public sector. From comparative analysis of publicly-produced health services in the best performing countries, we extract eight characteristics which contribute significantly to good performance. A final reflection is on the relevance of the importance of offsetting the potential hostility to a reformist impulse of the status-quo with alliances that strengthen public trust and the social contract between health professionals and citizens based on the values of public health systems.

Key words Good governance, Publicly-produced health services, $M$ anagement, International comparisons, Contractual management, $\mathrm{N}$ ew public service
Resumen El buen desempeño de servicios de salud de producción pública tiene importancia vital, más allá del sector salud. Se aborda su buen gobierno y gestión a partir del marco conceptual de los valores e ideas del buen gobierno y de la experiencia de países más ejemplares, como referente práctico de buen desempeño. Se parte de la gran complejidad de los servicios públicos de salud, por su carácter público y su naturaleza profesional, y se identifican siete nudos gordianos de los servicios públicos de salud en España e I beroamérica. Del concepto de buen gobierno se toma su carácter de referente ético y normativo y su potencial para renovar y dinamizar el gobierno delo público. Del análisis comparado de los servicios de salud de producción pública se extraen ocho características que contribuyen a su buen desempeño. Se concluye con una reflexión sobre la importancia de compensar la posi ble hostilidad corporativa y gremial a un impulso reformista del statu-quo con alianzas que refuercen la confianza pública y el contrato social entre profesionales y ciudadanos en torno a los valores de los sistemas públicos de salud.

Palabras clave Buen gobierno, Servicios sanitarios de producción pública, Ggestión, Comparación internacional, Gestión contractual, Nuevo servicio público 


\section{Introducción}

El buen gobierno de los intereses públicos es uno de los principales retos de las sociedades de todas las épocas. En cada sociedad y momento histórico las tradiciones, la cultura, la religión y el juego de intereses han ido acuñando respuestas institucionales para gobernar y gestionar lo público. El Estado democrático de los países desarrollados es la manifestación más avanzada de esta evolución. Dentro del mismo, coexisten sin embargo en tensión permanente diferentes esquemas conceptuales sobre el papel del Estado, su esfera de actuación y los instrumentos de la misma. Estas concepciones reflejan diferentes perspectivasideológicas y/o los de los actores implicados en las diversas opciones políticas, institucionales y organizativas existentes. La actual crisis económica ha contribuido a hacer visible la desnudez del emperador, los efectos negativos de las políticas desmanteladoras del Estado. Así, los premios Nobel Krugman ${ }^{1}$ y Stiglitz ${ }^{2}$ atribuyen en gran medida la actual crisis económicaal debilitamiento de las instituciones del Estado encargadas de regular y controlar los mercados financieros.

Este trabajo trata del gobierno de los servicios de salud. Asume de partida que su financiación (por impuestos y/o seguros sociales obligatorios) eslaúnica alternativa equitativa, racional y eficiente de aseguramiento-financiación. No entra en el debatesobreventajas/inconvenientes dela producción pública integrada delos servicioso decontratación externa ${ }^{3}$. Su punto de partida es una doble constatación: (1) que la inmensa mayoría de los servicios públicos de salud en España, Portugal y gran parte de Iberoamérica son de producción pública directa (gobiernos regionales o centrales), (2) que su desempeño en eficiencia y calidad dista mucho deser satisfactorio. El objetivo del presente trabajo es ofrecer un conjunto consistentedealternativas para el buen gobierno y la buena gestión de los servicios públicos desalud, a partir dela potencialidad e implicaciones del concepto de buen gobierno, de sus valoresy principios, aplicados alos instrumentos degobierno corporativo de lo público, así como de las buenas prácticas de aquellos países que cuentan con servicios ejemplares de salud de producción pública, en los que la gestión contractual desempeña un papel particularmente útil. No se trata aquí del conjunto del sistema de salud (nivel "macro"), si no únicamente del buen gobierno y gestión de las organizaciones, centros y unidades operativas que prestan servicios directamente a la población (Distritos o Áreas de Atención Primaria, H ospitales, etc.).
Para ello, analizaremos las raíces de la gran complejidad de los servicios públicos de salud, exploraremos el concepto de buen gobierno y extraeremos las enseñanzas que se derivan de la experiencia de los servicios públicos de salud de los países más avanzados en buen gobierno corporativo. Concluimos con una reflexión final a favor del desarrollo de un paradigma del "Nue va Gestión del Servicio Público" que consideramos elemento decisivo para el futuro de los servicios de salud de producción pública.

El reto de gobernar

y gestionar los servicios publicos de salud

La complejidad de los servicios públicos de salud viene dada, (1) por tratarse de un servicio prestado en ámbito de lo público, (2) por la naturaleza profesional de la M edicina y (3) por el contexto institucional y político.

En primer lugar, el Estado y la gestión de lo público tienen características muy específicas derivadas deoperar en marco jurídico y político que le da poder de coacción y autoridad legitimada por el proceso político democrático, y que a su vez le impone restricciones y controles a su organización y a su autonomía queson esenciales para la transparencia y rendición de cuentas 4 propias de un Estado democrático. Estas características fueron diseñadas fundamentalmente para impedir el ejercicio arbitrario del poder; muchas datan del Estado del siglo XIX. No están pensadas no para buscar la eficiencia que se exige a un Estado moderno, proveedor deservicios públicos. Larespuesta organizativa de los Estados a estas nuevas necesidades ha sido diferente en cada uno de los dos grandes prototipos de culturas político-administrativas. En la cultura política anglosajona (Reino Unido, Estados Unidos, Canadá, Australia, Nueva Zelanda), dada su orientación más gerencialista, su mayor flexibilidad y orientación a objetivos más que a procedimientos, esta evolución parece haber sido más natural. Seexpresa en el paradigma de la Nueva Gestión Pública ( New Public M anagement) ${ }^{5}$, que ha dominado la literatura internacional sobre estos temas.

Por el contrario, en los Estados de tradición administrativa napoleónica ${ }^{6}$ (Francia, España, Portugal y toda I beroamérica) la administración pública está más diseñada para la objetividad y la prevención de abusos, corrupción y fraudes, a través del control del cumplimiento de las normas. Su gestión se basa en procesos reglados en los cuales el operador público tiene un margen 
muy limitado de discrecionalidad, con un control exhaustivo "ex ante" de todas sus decisiones, centrado en el cumplimiento denormas y procedimientos, más que en la eficiencia o efectividad. En estos países los nuevos servicios públicos del Estado moderno han dado lugar al desarrollo de organizaciones, cuerpos funcionariales y normas específicas dentro de su administración pública tradicional. Sin embargo, con la excepción parcial deFrancia, país de referencia deesteparadigma, todos los países de este grupo presentan, en mayor o menor grado, problemas y notables dificultades para lograr en los servicios públicos los niveles de eficiencia y calidad que piden sus ciudadanos. Un problema no menor para ello es la escasez en el mercado de ideas de un paradigma de N ueva Gestión del Servicio Público ${ }^{7}$ ( $\mathrm{N}$ ew Public Service), equivalente al de la Nueva Gestión Pública, que tome como punto de partida los valores de la buena administración pública tradicional, para buscar su modernización y su mayor eficienciå.

La segunda fuente de complejidad en la gestión delos servicios públicos desalud deriva dela naturaleza profesional de la práctica médica, que hace de los hospitales el prototipo clásico de organización profesional ${ }^{9}$, donde la materia prima esel conocimiento de unos profesionales, queejercen su actividad con autonomía respecto a la jerarquía de la organización. Ésta detenta el poder administrativo pero no la autoridad profesional, la cual por estar basada en el conocimiento, pertenece a los profesionales ${ }^{10}$. Ello conlleva retos muy específicos de gestión y gobierno, con tensiones clásicas entre administración y profesionales, aunque lo verdaderamente crítico para la eficiencia y calidad de los servicios de salud es la vigencia práctica de los valores, principios y compromisos del profesionalismo médico ${ }^{11}$.

El tercer elemento que determina la extraordinaria complejidad del gobierno y gestión de los servicios públicos de salud es el contexto institucional y social concreto del sector público de cada país. Su cultura y sus prácticas políticas, administrativas y de gestión prolongan en los servicios públicos de salud (y otros) todas sus dimensiones, positivas o negativas. Un Estado con un desempeño global eficaz tendrá servicios públicos de salud eficaces, y viceversa: la corrupción y la ineficiencia del sector público inevitablemente se verá reflejada en los servicios públicos de salud.

\section{La idea del buen gobierno}

El concepto debuen gobierno. La expresión "buen gobierno" contiene un juicio de valor ("bueno") y un concepto complejo y polisémico ("gobierno"), que en este contexto, se refiere al modo de gobernar, también conocido como "gobernanza" (en inglés, governance). Es un concepto que ha adquirido una gran relevancia en las últimas décadas, sobre todo por la prioridad que ha recibido por parte de las grandes organizaciones internacionales, especialmente el Banco Mundial ${ }^{12}$, pero también la OM S, PNUD ${ }^{13}, \mathrm{OCDE}^{14}$, etc. La expresión "buen gobierno" (good governance) no tiene una definición estándar y, como sucede con la salud, frecuentemente se mide a partir de sus contrarios: corrupción, despilfarro, nepotismo, ineficiencia (Banco Mundial ${ }^{15}$ y Transparencia Internacional $\left.{ }^{16}\right)$.

Pero, además de prevenir la corrupción y lograr un alto nivel de "integridad", el buen gobierno persigue otros objetivos del máximo interés social, esenciales para que las organizaciones sanitarias cumplan su misión social: calidad, eficiencia, transparencia, rendición decuentas, participación democrática, etc. El buen gobierno, exige buenos "resultados" ( efectividad, eficiencia, calidad, equidad), pero también buenos "procedimientos" detoma dedecisiones, deacuerdo a "normas" de participación democrática, de transparencia, de rendición de cuentas, de respeto a las leyes, etc. Procedimientos que están basados en "valores" deética pública y política, queno es sino ética pública aplicada al gobierno y a la gestión de lo público, a la Administración y a todos sus servicios. Una ética política es obligada en todo el sector público, e incluye a los proveedores deservicios públicos, para los cuales es partedesu ética profesional. Sus principios y reglas, son entre otros: respeto a la ley, búsqueda del interés público, transparencia y rendición de cuentas, eficiencia, equidad, igualdad de trato y oportunidades, participación ciudadana y búsqueda del consenso, regulación de los conflictos deinterés, etc. Buen gobierno es, pues, calidad institucional ${ }^{17}$. No cabe hablar de "buen gobierno" si falta alguno de los eslabones de la cadena causal queliga valores (éticos y democráticos), "procedimientos" de decisión eficaces que respondan a esos valores, y finalmente, "resultados" positivos.

Estas reflexiones son aplicables al sector salud ${ }^{18}$, como hace el Consejo de Europa en sus Recomendaciones para el Buen Gobierno de los Sistemas de Salud ${ }^{19}$ (Cuadro 1). La premisa que subyaceen sus recomendaciones es que una bue- 
na gobernanza basada en valores, influencia positivamente todas las funciones del sistema sanitario, mejorando su desempeño y, en última estancia, los resultados de salud.

\section{Buen gobierno corporativo} de los servicios públicos de salud

El modo decontrolar y dirigir una organización se conoce como gobierno corporativo, una expresión nacida en el sector privado, donde es de uso común. Sin embargo, cada vez es más utilizada en el sector público (public sector governance) espe cialmente en los países de cultura anglosajona, destacando en ello Australia y Nueva Zelanda ${ }^{20}$.

El gobierno corporativo de una organización se refiere a las estructuras de la misma, a los procedimientos existentes para lograr una gestión efectiva y eficiente, y a los mecanismos con los que se busca el cumplimiento de normas y objetivos y se previenen conductas contrarias a los mismos. La Australian National Audit Office $(A N A O)^{21}$ define la gobernanza en el sector público como los procesos a través de los cuales se dirige, controla y se ejerce la rendición de cuentas de una organización. Posteriormente esta definición ha sido expandida para incluir también la cultura, la estrategia y las políticas de la organización, así como la forma en la que se relaciona con todos los actores implicados 22 .

Un punto importantepara enten der el gobierno corporativo es diferenciar en las organizaciones gobierno y gestión: gestionar (gestión) es diferente a gobernar (gobernanza). De la función de gobierno emanan las estructuras, normas y estrategia querigen la organización, con lascuales operael gestor; la función deéstees la deejecutor, es decir: ser responsable del día a día, y rendir cuentas de su gestión al órgano colegiado de gobierno (Patronato, Consejo de Gobierno o de Administración). Esta distinción, tradicional en muchasinstituciones públicas especialmentehospitales, frecuentementeha quedado devaluada por la pérdida del papel que corresponde a los órganos degobierno. Estepunto es crucial, porqueun argumento central de este trabajo es que la recuperación de la función gobierno de los órganos colegiados tieneun gran potencial para renovar la gestión delas organizaciones públicas de servicios de salud y mejorar su desempeño.

Los principios del buen gobierno corporativo son similares en el sector público y en el privado: rendición de cuentas (accountability), transparencia, integridad, respecto a las reglas, eficiencia, efectividad, calidad, respetar los derechos de terceros (afectados, implicados, etc.). Es deespecial interés práctico conocer los instrumentos (estructuras y procedimientos) del buen gobierno corporativo que contribuyen a un desempeño óptimo de las organizaciones. Del conjunto de códigos existentes, tanto para el buen gobierno corporativo del sector privado ${ }^{23}$ como del públi$\mathrm{CO}^{24,25}$, se pueden extraer los elementos básicos mostrados en el Cuadro 2.

De estos elementos del buen gobierno corporativo, los órganos colegiados de gobierno (los Consejos) son sin duda el componente más importante, pero al mismo tiempo el más frágil. Su importancia deriva de ser el vértice de autoridad de sus organizaciones, de su potencial para tener una gran legitimidad social y política, por las posibilidades queda su carácter colectivo, y por ofrecer mayores garantías que un único órgano uni-

Cuadro 1. Consejo de Europa. Recomendación CM/Rec(2010) 6 sobre el buen gobierno de los sistemas de salud, 2010.

\begin{tabular}{|l|}
\hline \multicolumn{1}{|c|}{ Apéndice (directrices) } \\
\hline I. Leyes y reglamentos para el buen gobierno de un sistema de salud basado en los valores y principios \\
fundamentales. \\
II. Promover códigos de conducta para la buena gestión de un sistema de salud. \\
III. Seguimiento de buen gobierno de un sistema de salud. \\
IV. Gestión de conflictos de interés en los sistemas de salud. \\
V. Una estrategia de buen gobierno contra el fraude y la corrupción. \\
VI. Preparación de los profesionales relacionados con la salud para el buen gobierno: la educación y la \\
formación de profesionales de la salud, administradores, gerentes y responsables de las políticas de buen \\
gobierno basado en los valores de los sistemas de salud.
\end{tabular}

Fuente: Consejo de Europa (Adopted by the Committee of M inisters on $31 \mathrm{M}$ arch 2010), www.coe.int 
Cuadro 2. Características básicas del buen gobierno corporativo de las organizaciones sanitarias.

1. Existencia de un órgano colegiado de gobierno (Consejo), diferenciado de la Dirección ejecutiva (órgano gestor).

2. Consejos con características que la experiencia ha demostrado que contribuyen a su efectividad: (a) compuesto por miembros sin poder ejecutivo en la organización e independientes, sin intereses personales en ella; (b) tamaño adecuado, (c) consejeros con competencias relevantes para la organización, (d) funcionamiento de un subcomité de auditoría y de otros subcomités formados por los consejeros según necesidades.

3. Dirección ejecutiva (o Dirección-Gerencia): (a) seleccionada por procesos transparentes, (b) retribución con incentivos por desempeño.

4. Reglas de funcionamiento del Consejo y de la dirección ejecutiva, así como de la relación entre ambos, que respondan a estándares de los códigos de buen gobierno y buena gestión.

Fuente: Autores, Códigos debuen gobierno.

personal al buen funcionamiento y al cumplimiento delosfines dela organización ${ }^{26}$. Dehecho, esla forma de gobierno de todas las organizaciones con una cierta complejidad en los diferentes países. Por otro lado, de nada sirve la existencia de Consejossi éstosno son efectivos, lo cual requiere formar a sus miembros y la existencia de reglas y

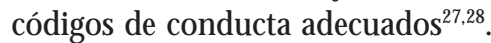

Aprendiendo de los mejores servicios de salud de produccion pública

Ocho características

de los buenos servicios públicos de salud

Las instituciones que determinan las estructuras y procedimientos de gobierno y gestión de las organizaciones públicas, son el resultado de una compleja evolución histórica, compuesta de múltiples capas y dimensiones y con una notable inercia (dependencia de senda). Dado el fuerte carácter contextual, las experiencias ajenas raramente pueden, o deben, ser copiadas de un entorno a otro, aunque siempre es obligado aprender de ellas, adaptándolas a la propia realidad. Sin embargo, las comparaciones ${ }^{29}$, han tenido y tienen una gran influencia en la construcción de políticas y en las propuestas de reformas.

Existien do países cuyos servicios de salud destacan claramente por su alto nivel de eficiencia, calidad y equidad (acceso universal equitativo), tiene gran interés preguntarse por las características comunes a todos ellos en su organización, gobierno y gestión y contrastar estas características con las de nuestros propios servicios públicos. El análisis comparado ${ }^{30}$ de los aspectos rele- vantes del gobierno corporativo (estructuras y mecanismos de gobierno y gestión) de los servicios públicos de salud se ve dificultado por la escasez en la literatura einformación sobre estos temas, tanto a nivel central, como territorial (Distritos, Áreas de Salud), con la excepción parcial de los hospitales.

Para nuestro análisis hemos considerado únicamente países con sistemas de servicios públicos de salud universales e integrados: Reino Unido, Italia, España, Países N órdicos (Dinamarca, Finlandia, Noruega y Suecia) y Portugal, y de fuera de Europa a Nueva Zelanda. La información ha sido extraída del European Observatory on Health Systems and Policies (cuya serie H ealth Systems in Transition HiT-31 contiene un informe detallado de cada país), de la literatura profesional ${ }^{32,33}$, de las páginas oficiales de Internet, así como de un estudio ad hoc realizado por los autores en cinco países europeos ${ }^{34}$.

Este grupo de países incluye las dos tradiciones de administración pública, la gerencialista anglosajona y la administrativa napoleónica, diferencia, que como cabría esperar, tiene una gran influencia en el gobierno corporativo de los servicios públicos de salud. En al gunos deestos países (Suecia, Reino Unido, España el talia) los servicios de salud son responsabilidad de los gobiernos subnacionales, en un contexto semifederal; ello da lugar en ocasiones a notables diferencias entrelas regiones de un mismo país (Escocia elnglaterra). El Cuadro 3 muestra ocho características presentes en los países analizados. $\mathrm{H}$ an sido sel eccionadas por considerarlas especialmente decisivas para el buen gobierno corporativo de los servicios públicos de salud. En nuestra opinión su existencia condiciona el buen desempeño 
deÁreas/Distritos de Salud y Hospitales. A continuación analizamos brevemente las características seleccionadas y las peculiaridades que presentan en algunos países.

Separación y diferenciación

entre el sistema sanitario público

y la administración pública general

Los modernos servicios de salud son muy específicos y su gobierno tieneexigencias muy diferentes a las de la administración pública común. Esta lógica lleva a dar a los servicios públicos de salud una personalidad jurídica diferenciada dela administración pública común. Es interesante constatar las dificultades en los países de tradición administrativa para adoptar formulas tipo empresa pública para sus servicios de salud; mientras que es fórmula general en Italia y en los hospitales portugueses, en España solo algunas Comunidades Autónomas la han adoptado para el conjunto de sus servicios de salud (País Vasco, Instituto Catalán de la Salud, Servicio M adrileño de Salud), pero en hospitales solo se utiliza en los de nueva creación. Estas reformas han encontra-

Cuadro 3. Características importantes de buen gobierno y desempeño en sistemas de salud públicos integrados de países seleccionados.

\begin{tabular}{|c|c|c|c|c|c|c|}
\hline & $\begin{array}{l}\text { Reino } \\
\text { Unido }\end{array}$ & $\begin{array}{l}\text { Países } \\
\text { nórdicos }\end{array}$ & $\begin{array}{l}\text { Nueva } \\
\text { Zelanda }\end{array}$ & Italia & España & Portugal \\
\hline $\begin{array}{l}\text { Los servicios públicos de salud tienen } \\
\text { personalidad jurídica propia, } \\
\text { diferenciada de la Administración } \\
\text { pública. }\end{array}$ & $\mathrm{Si}$ & $\mathrm{Si}$ & $\mathrm{Si}$ & $\mathrm{Si}$ & $\mathrm{Si}$ & $\mathrm{Si}$ \\
\hline $\begin{array}{l}\text { Organización territorial descentralizada } \\
\text { (Áreas/Distritos/hospitales). }\end{array}$ & $\mathrm{Si}$ & $\mathrm{Si}$ & $\mathrm{Si}$ & $\mathrm{Si}$ & $\mathrm{Si}$ & $\mathrm{Si}$ \\
\hline $\begin{array}{l}\text { Gobierno de Áreas/Distritos/hospitales } \\
\text { en base a órganos colegiados de } \\
\text { gobierno (Consejos) ante los que rinden } \\
\text { cuentas los gestores. }\end{array}$ & $\mathrm{Si}$ & $\mathrm{Si}$ & $\mathrm{Si}$ & No & No & No \\
\hline $\begin{array}{l}\text { Los gestores tienen carácter profesional, } \\
\text { no son nombramientos discrecionales } \\
\text { (políticos). }\end{array}$ & $\mathrm{Si}$ & $\mathrm{Si}$ & $\mathrm{Si}$ & No & No & No \\
\hline $\begin{array}{l}\text { Alto nivel de autonomía, tanto a nivel } \\
\text { central respecto a la administración } \\
\text { general, como localmente (Áreas/ } \\
\text { Distritos/Hospitales), que incluye } \\
\text { contratación del personal propio. }\end{array}$ & $\mathrm{Si}$ & $\mathrm{Si}$ & $\mathrm{Si}$ & $\mathrm{Si}$ & No & $\mathrm{Si}$ \\
\hline $\begin{array}{l}\text { Atención Primaria fuerte: M édico } \\
\text { General/de Familia puerta de entrada } \\
\text { para la atención especializada, con lista } \\
\text { de población a cargo. }\end{array}$ & $\mathrm{Si}$ & No (Suecia) & $\mathrm{Si}$ & $\mathrm{Si}$ & $\mathrm{Si}$ & Si \\
\hline $\begin{array}{l}\text { Regulación y organización de la } \\
\text { profesión médica (y otras profesiones de } \\
\text { la salud) favorable a los valores de la } \\
\text { profesionalidad, al diferenciar } \\
\text { regulación, aspectos sindicales y temas } \\
\text { profesionales (conocimiento- } \\
\text { competencias). }\end{array}$ & $\mathrm{Si}$ & $\mathrm{Si}$ & $\mathrm{Si}$ & No & No & $\mathrm{Si}$ \\
\hline $\begin{array}{l}\text { Desarrollo de la gestión contractual en } \\
\text { cascada (intraorganizativa, o entre } \\
\text { financiadores y proveedores) hasta el } \\
\text { nivel de unidades clínicas. }\end{array}$ & $\mathrm{Si}$ & $\mathrm{Si}$ & $\mathrm{Si}$ & $\mathrm{Si}$ & $\mathrm{Si}$ & No datos \\
\hline
\end{tabular}


do resistencias, incluido el sorprendentereproche de "privatización"; el inmovilismo en lo administrativo alimenta dehecho la externalización hacia modelos privados comerciales.

Organización descentralizada

de los servicios con base territorial

La planificación poblacional y territorial de los recursos en Áreas o Distritos de Salud, por razones de racionalidad y equidad, precisa deuna organización de los servicios descentralizada en el territorio. Es esencial además para una buena gestión, con autonomía y control democrático, así como para la coordinación de los servicios y una atención integrada. Esta importante característica es común a todos los países anualizados, sin excepción.

Gobierno en base a órganos colegiados de gobierno (Consejos) ante los que rinden cuentas los gestores

En estos Consejos, de Áreas/Distritos de Salud o de los hospitales reside la máxima autoridad de la organización; son un cauce de participación democrática y control cívico de los servicios públicos, que se contrapone al órgano unipersonal de gestión, parte de una cadena jerárquica de mando. El gobierno a través de Consejos es "la norma en todo tipo de organizaciones complejas". El Cuadro 3 muestra en este punto las diferencias entre países con distintas tradiciones político-administrativas.

Gestores profesionales

Seleccionados con publicidad y transparencia, en base a su capacidad y experiencia, que responden antelos Consejos degobierno. El carácter profesional desu función, la ausencia de connotación partidariay la rendición decuentas antelos Consejos confierea los gestores la legitimidad, autoridad y estabilidad esenciales para su buen desempeño. También en este punto existe un contraste claro entrelos países pertenecientesalamisma tradición político-administrativa: en España los responsables de los servicios públicos de salud y de sus instituciones (hospitales por ejemplo) tienen actualmente la consideración de cargos de confianza, de nombramiento político; en Francia sin embargo, los directores de hospitales públicos tienen un carácter funcionarial-meritocrático.

Alto nivel de autonomía de gobierno y gestión

Dentro un marco común, tanto en el nivel central respecto a la Administración general), como localmente, las Áreas/Distritos de Salud, hospitales. Todos los países anal izados tienen un alto grado de autonomía local, esencial para el buen funcionamiento de los servicios. Esta autonomía local, que opera dentro del marco corporativo general de los servicios públicos de salud, incluyela contratación por los centros de su propio personal, en condiciones muy similares cuando no idénticas como en los países nórdicos- a las cualquier otro trabajador.

Atención Primaria fuerte

Se manifiesta principalmente, aunque no exclusivamente, en el papel de M édico General/de Familia como puerta de entrada a la atención especializada y en la continuidad que supone tener establemente una lista de población a su cargo. No es posible insistir bastante en que la buena organización y funcionamiento de este primer nivel de atención es condición esencial para la eficiencia, calidad y equidad (acceso universal) de los sistemas de salud. De los países analizados, la única excepción - más teórica quereal por otra parte- es Suecia donde los pacientes pueden acudir directamente al hospital. España, destaca en este punto por la tradición y la solidez de su Atención Primaria.

Regulación y organización de la profesión médica (y otras profesiones de la salud)

La naturaleza profesional de los servicios de salud, hace particularmente importante un buen marco regulatorio y organizativo de la profesión médica y otras profesiones sanitarias, que favorezca y promueva los valores del profesionalismo. Ello implica diferenciación y organización eficaz de tres elementos: (1) la regulación legal, que corresponde al Estado, y que éste puede delegar parcialmente, (2) la gestión del conocimiento y competencias profesionales, actividad central de los Colegios u Ó rdenes y (3) la defensa de los intereses, responsabilidad propia de los sindicatos. Este esquema es típico de los países de tradición anglosajona y de los países nórdicos, y solo existe en parte en Francia, España, Italia y Portugal. Existen sin embargo líneas de convergencia y, por ejemplo, es de gran interés el desarrollo en España por la Organización M édica Colegial, deun amplio consenso profesional para impulsar los valores del profesionalismo, vinculándolo a la responsabilidad de los médicos hacia el Sistema Nacional deSalud ${ }^{35}$. 
Desarrollo de la gestión contractual

pública, intraorganizativa o entre

financiadores y proveedores públicos

Los fundamentos profundos de este desarroIlo tienen que ver con la búsqueda de herramientas que complementen el modelo jerárquico dominante en el ámbito administrativo (eintra-organizativo, en general), habida cuenta desu insuficiencia real para gobernar organizaciones profesionales como son las desalud. En ellas el recurso a la estandarización no es suficiente, ni en su versión industrial (Tayloriana), ni en su versión procedimental (burocrático-Weberiana de clara inspiración napoleónica). De ahí la búsqueda de instrumentos nuevos que dentro de la arquitectura organizativa pública permitan obtener ganancias degobernabilidad en la función sanitaria. Los contratos de gestión o contratos-programa, suscritos entre el financiador público y los proveedores públicos, son uno de los experimentos desarrollados en países con modelos sanitarios integrados en los años 90 y presentan aspectos positivos derelevancia ${ }^{36}$. Setrata deun documento, que remeda un contrato, complementando la relación jerárquica o intra-organizativa, cuya base de autoridad está alterada en las organizaciones profesionales. Este tipo de instrumentos los contratos facilitan la implicación de los profesionales y la visualización delos compromisos de recursos por actividad, calidad y resultados.

El hecho de que los servicios de salud que se desempeñan con mayor nivel de calidad, eficiencia y equidad (acceso universal) son los de aquellos países que también tienen instituciones de más calidad, más sólidas y legitimadas, mejores indicadores de buen gobierno, apunta claramente a la importancia del contexto y de la calidad dela política ${ }^{37}$. Las ocho características indicadas arriba, si bien valiosas por sí mismas, solo desarroIlan todo su potencial de buen gobierno en un contexto político-social con un alto nivel detransparencia, rendición de cuentas, participación democrática y, en definitiva: una cultura de ética cívica y de servicio público. Sin embargo, es precisamente en ausencia de este entorno favorable cuando son más necesarias por su valor para inducir cultura de buena gestión pública y por ser instrumentos imprescindibles para la misma.

Es interesante comprobar cómo en los países detradición administrativa napoleónica quetra- tan de mejorar el desempeño de sus servicios públicos de salud, se reproducen los mismos temas y debates en relación a la imprescindible mejora de sus estructuras de gobierno y gestión. En España $a^{38}$ desde los años 90 , y más recientemente con la conversión del Instituto Catalán de Salud (ICS) en empresa pública; en Chile en relación a la autonomía de sus hospitales públicos ${ }^{39}$; en Portugal con la transformación de sus hospitales en entidades públicas empresariales (EPE) ${ }^{40}$. Aunque este tipo de reformas - tan necesarias por otro lado- encuentran una notable resistencia, seestán produciendo avancesen la práctica, como la reciente el Código de Buen Gobierno para la Sanidad Pública Vasca ${ }^{41}$ y los avances la gestión contractual.

\section{Reflexión final}

La mejora de los centros sanitarios públicos de gestión directa esun objetivo irrenunciabley prioritario para los sistemas sanitarios. Para ello se precisan cambios en su gobierno y en la gestión. El concepto deBuen Gobierno, aplicado al ámbito de la meso-gestión nos señala la importancia estratégica del gobierno corporativo, del queexisten experienciasinternacionalesútiles, y quetiene su concreción fundamental en la creación y adecuado funcionamiento de órganos colegiados de gobierno (Consejos). Por su parte, la mejora de la gestión es más factible con de la profesionalización de los directivos, con la gestión contractual y con todo un conjunto de políticas que permitan estimular el profesionalismo, mejorar la trasparencia y facilitar la rendición de cuentas.

En el mundo actual, los valores sobre los que se asientan los sistemas públicos de salud deben necesariamente concretarseen cambios reformistas y regeneracionistas en los hospitales y otros centros sanitarios públicos. La hostilidad corporativa y gremial quepodría suscitar el cambio del status-quo debe ser compensada con la transparencia y rendición de cuentas queimplica el buen gobierno y con alianzas que refuercen el contrato social entre profesionales, pacientes y sistemas de salud. El desarrollo de una cultura de "Nueva Gestión del Servicio Público" esun elemento decisivo para el futuro de los servicios de salud de producción pública. 


\section{Colaboradores}

J-M Freire y JR Repullo concibieron conjuntamente la idea del trabajo. JM Freire escribió un primer borrador que JR Repullo completó. Ambos autores revisaron complementariamente diferentes versiones del manuscrito y se corresponsabilizan por igual dela versión final.

\section{Referencias}

1. Krugman P. The conscience of a liberal. New York: Norton; 2009.

2. Stiglitz J. El papel económico del estado. Madrid: Ministerio de Economía y Hacienda,. Instituto de Estudios Fiscales; 1993.

3. Preker AS, Harding A, Travis P. Make or buy decisions in the production of health care goods and services: new insights from institutional economics and organizational theory. Bulletin of the World Health Organization 2000; 78:779-790.

4. Jiménez Hernández $\mathrm{E}$. Reinterpretando la rendición de cuentas o accountability: diez propuestas para la mejora de la calidad democrática y la eficacia de las políticas públicas en España [Internet]. 2009. [ consultado 2010 nov 28]. Disponible en: http:/ /bit.ly/hG2f9a

5. Pollitt C. What can we learn from 30 years of public management reform? [Internet]. Rome: 2010. [cited 2010 nov 28]. Available from: http://www. sspa.it/ wp-content/uploads/2010/11/Pollitt_ROME 20102.pdf

6. Ongaro E. Public management reform and modernization: trajectories of administrative change in Italy, France, Greece, Portugal and Spain. Chetelham: Edward Elgar Publishing; 2010.

7. Denhardt RB, Denhardt JV. The new public service: serving rather than steering. Public Administration Review 2000; 60(6):549-559.

8. Lynn Jr. LE. The myth of the bureaucratic paradigm: what traditional public administration really stood for. Public Administration Review 2001; 61(2): 144-160.

9. Etzioni A. Modern organizations. Englewood Cliffs, N.J.: Prentice-Hall, 1964. (Traducción española: M exico City: U.T.E.H.A., 1965).

10. Varela J, Craywinckel G, Esteve M, Pica JM. Implicación de los médicos en la gestión basa en el profesionalismo y en el liderazgo de equipos multidisciplinarios. Med Clin 2010; 134(1)35-39.

11. Borrell-Carrio F, Epstein RM, Pardell Alentà H. Profesionalidad y professionalism: fundamentos, contenidos, praxis y docencia. M ed Clin (Barc) 2006 sep 9; 127(9):337-342.

12. World Bank. Reforming public institutions and strengthening governance: a world bank strategy. Washington, DC: World Bank, Poverty Reduction and Economic M anagement Network; 2000.

13. United Nations Development Program (UNDP). Governance for sustainable human development. N ew York: UNDP; 1997.

14. OECD. Principal elements of good governance [Internet]. [cited $2010 \mathrm{Dec} 10]$. Available from: http:// www.oecd.org/document/32/0,3746,en_2649_ 3373518145601111 1,00.html

15. El BM tiene una gran riqueza de recursos dedicados al Buen Gobierno. Gobernanza y Control de Corrupción. [cited 2010 dec 10]. Available from: www.worldbank.org/wbi/governance/esp

16. Transparency International España. Barómetro Global de la Corrupción. [Internet]. [cited 2010 Dec 10]. Available from: http://www.transparencia.org.es/ 
17. Chong A, Gradstein M. Institutional quality and government efficiency [Internet]. Inter-American Development Bank; 2007. [cited 2010 Dec 10]. Available from: http://idbdocs.iadb.org/wsdocs/getdocument. aspx?docnum $=35061567$

18. Ortun V, editor. El buen gobierno sanitario. Madrid: Springer Healthcare Communications; 2009.

19. Council of Europe-Committee of Ministers. Recommendation CM/Rec(2010) 6 of the Committee of $M$ inisters to member states on good governance in health systems. European $\mathrm{H}$ ealth Committee. [cited 2010 dec 10]. Available from: http://bit.ly/hWVDWE

20. Corporate Governance ARC Project. University of Canberra, Australia [Internet]. [cited 2010 Dec 8]. Available from: http://www.canberra.edu.au/corp gov\%2Daps/

21. Australian National Audit Office. Corporate governance in Commonwealth authorities and companies: principles and better practices. Canberra: Australian National Audit Office; 1999.

22. Edwards M, Clough R. Corporate governance and performance: an exploration of the connection in a public sector context. Canberra: University of Canberra; 2005. [cited $2010 \mathrm{Dec} 10$ ]. Available from: http://bit.ly/hBiNag

23. Organisation for Economic Co-operation and Development. OECD principles of corporate governance. Paris: OECD; 2004. [cited 2010 Dec 10]. Available from: www.oecd.org/daf/corporate/principles

24. Victorian Government (Australia). State Services Authority. Good practice guide: reference list [Internet]. [cited $2010 \mathrm{Dec} 10$ ]. Available from: http:// www.ssa.vic.gov.au/CA2571C900701857/0/91471A81 AD940C54CA2571CB002B9A7A

25. The Independent Commission on Good Governance in Public Services. The good governance standard for public services [Internet]. London: OPM (Office for Public Management) and CIPFA (The Chartered I. of Public Finance and Accountancy); 2004. [cited 2010 Dec 10]. Available from: www.cipfa. org.uk

26. Bennington L. Review of the corporate and healthcare governance literature. Int J H ealth Plann M anage 2010; 16(2):314-333.

27. Kathryn $\mathrm{O}^{\prime} \mathrm{N}$ eill J, editor. Putting quality first in the boardroom: improving the business of caring. London: The King's Fund; 2010.

28. NHS Healthy Board Principles Document. The National Leadership Council [Internet]. [cited 2010 Oct 13]. Available from: http://www.nhsleadership. org.uk/workstreams-board-nhshealthyboard.asp

29. Conill E. Sistemas comparados de saúde. In: Campos GW, M inayo M CS, Akerman M, organizadores. Tratado de Sauide Coletiva. Sapo Paulo: Hucitec; Rio de Janeiro: Editora Fiocruz; 2006. p. 563-615.

30. James 0 , Wilson $D$. Introduction: evidence from the comparison of public service performance. Evaluation 2010; 16(1):5-12.
31. WHO/Europe. Health system profiles (HiTs) [Internet]. [cited 2010 Dec 10]. Available from: http:// www.euro.who.int/en/home/projects/observatory/ publications/health-system-profiles-hits

32. Dickinson H, H am C. H ealth Services in Small Countries: what are the lessons for Wales? [Internet]. 2008. [cited 2010 Dec 10]. Disponible en: http:// www.hsmc.bham.ac.uk/news/pdfs/Governance_ Small_Country_English.pdf

33. Dentzer S. Lessons on health reform from across the waves. Health Affairs 2010; 29(8):1428.

34. Repullo J, Freire J, editores. M édicos en cinco Sistemas Sanitarios Integrados Europeos: relaciones contractuales de los profesionales médicos en los sistemas públicos de salud en países europeos seleccionados. [Internet] 2009. [consultado $2010 \mathrm{~N}$ ov 28]. Disponible en: http://bit.ly/gQ e5yk

35. Rodriguez Sendín J. Los compromisos de la profesión médica ante los desafíos actuales. Conferencia Siglo XXI. M adrid: 2010. [consultado 2010 Nov 18]. Disponible en: http://www.cgcom.org/sites/default/ files/Conferencia.doc

36. Øvretveit J. Purchasing for health. Buckingham: O pen University Press; 1995.

37. Bresser-Pereira L, Grau N, Garnier L, Oszlak O, Przeworski A, editores. Politica y gestión pública. Buenos Aires, Caracas: Fondo de Cultura Económica, CLAD; 2004

38. $M$ artin $M$ artin J. El gobierno de las organizaciones sanitarias del estado español. In: Repullo Labrador J, Oteo Ochoa L, editors. Un nuevo contrato social para un sistema nacional de salud sostenible. BarceIona: Editorial Ariel; 2005. p. 333-348.

39. M éndez CA,Torres AM C. Autonomía en la gestión hospitalaria en Chile: Ios desafíos para el recurso humano en salud. Rev Saude Publica 2010; 44(2):366371.

40. Campos A. Reformas da sauide: o fio condutor. Coimbra: Almedina; 2008.

41. Recomendaciones del Consejo Asesor sobre el Buen Gobierno de la Sanidad Pública Vasca. [Internet]. [consultado $2012 \mathrm{Abr}$ 14]. Disponible en: http:// bit.ly/ihG bo8

Artigo apresentado em 23/02/2011

Aprovado em 30/03/2011

Versão final apresentada em 14/04/2011 